\title{
Macromolecular Structure and Organization of Alpha Keratin
}

\author{
P. J. HARGET* and S. KRIMM, Biophysics Research Division and \\ Harrison M. Randall Laboratory of Physics, University of Michigan, \\ Ann Arbor, Michigan 48104
}

\begin{abstract}
Synopsis
The small-angle equatorial X-ray scattering of alpha keratin (African porcupine quill) was studied using a direct type of analysis, which allows the separation of the intermicrofibrillar interference effects from those of the intramicrofibrillar scattering without the use of prior assumptions. X-ray data were obtained from $470-21 \AA$; their analysis by the above method resulted in a $z$-axis projection of the microfibrillar electron density, which extends over a diameter of $80 \AA$ and has a core region surrounded by a peak located at a radius of $28 \AA$. The macromolecular organization of the 80 - $\AA$-diameter microfibrils was found to be an arc-like layer type of aggregation and not a pseudohexagonal packing. When the specimen was subjected to silver staining the aggregation of the microfibrils did not change except for a slight contraction. The $z$-axis projection of the stained microfibril, however, was significantly different. The silver appears to build up slightly around the outer periphery of the microfibril, stains the peripheral region very little, deposits on the inside of the peripheral region, and outlines the core area.
\end{abstract}

\section{INTRODUCTION}

It was evident from early electron microscopy studies of alpha keratin ${ }^{1-3}$ that, almost irrespective of speciment type, this protein is composed of 7080 - $\AA$-diameter fibrous units called microfibrils embedded in a matrix. Later, Filshie and Rogers ${ }^{4}$ appeared to resolve units of the microfibril which were about $20 \AA$ in diameter. These subunits, or protofibrils, form the periphery of the microfibril and are separated by an interstitial region from a central core region consisting of one or two protofibrils. Subsequent studies $^{5,6}$ suggested that the 20 - $\AA$ subunits might be artifacts, but the ringcore structure was substantiated. This was also the case from other work. ${ }^{7}$

Progress in the analysis of X-ray data on alpha keratin has been difficult because of the poor degree of lateral order between microfibrils and the high degree of distortion present both within and between the microfibrils. Nevertheless, the wide-angle X-ray pattern yielded the alpha helix and the two- or three-stranded twisted rope (coiled coil) model for the protofibril..$^{8,9}$

In addition to the wide-angle region, where the protofibril dominates the scattering, there is another interesting scattering region. This is the small-

* Present address: Allied Chemical Corporation, Chemical Research Center, Morristown, N.J. 07960. 
angle equatorial scattering region, which, for African porcupine quill, has maxima located at $87 \AA, 45 \AA$, and $27 \AA$. The positions of these maxima, their relative intensities, and their shapes depend a great deal on the specimen or species from which the alpha keratin is obtained. ${ }^{10}$ Further, this region is strongly affected by different heavy metal stains in various ways. ${ }^{10,11}$ One of the general effects of heavy metals is to intensify this region relative to the high-angle region: intensification by as much as 25 times has been achieved with silver stain. Thus ${ }^{10}$ since silver is deposited in the matrix and somewhat in the microfibril, the scattering in this region is a function of a) the intermicrofibrillar interference function, b) the relative electron density of the interstitial material both inside and outside the microfibril, and c) the protofibril electron density and location in the microfibrils. The problem has been to separate these three effects from a single scattering curve and to evaluate them individually. Only in this way can structural details of the organization of protofibrils and microfibrils be obtained.

The attempts in the past to explain the scattering in this region have usually depended on assumed models for the structure. Since the microfibril measures about 70-80 $\AA$ in diameter in electron micrographs, Fraser and MacRae ${ }^{12-14}$ assumed that the transform of the microfibril in this region was simply that of a solid cylinder of radius $37 \AA$. They concluded further that, although the $87 \AA$ peak was caused by the intermicrofibrillar interference function, the 45 and $27 \AA$ peaks were caused by the secondary maxima of this solid cylinder transform. After evidence for the protofibril was presented, Wilson ${ }^{15}$ made a semiquantitative analysis, through model building, of the scattering in this small-angle region. He also noted that one would expect to find regions in any specimen with different numbers of microfibrils diffracting coherently, but he made no attempt to consider possible distributions, and simply assumed three microfibrils to be scattering coherently. For the microfibril structure he used nine $20 \AA$-diameter solid cylinders on the periphery and various numbers for the core. He was later criticized $^{16}$ for ignoring the possibility that relative electron density differences existed in the interstitial material, and also for comparing the results of his model with higher angle diffraction data. Later, Woods and Tyson, ${ }^{17}$ on the basis of the behavior of the three small-angle maxima with various metal strains, concluded that the true nature of this scattering derives from a structure somewhere between the model of Fraser and MacRae and that of Wilson, with the addition that electron density differences between protofibril and matrix, both internal and external to the microfibril, must be taken into account. Fraser, et al. ${ }^{18}$ departed from the model approach, basing their analysis only on the assumption of a rotation axis in the microfibril.

There is still another region of scattering on the equator which is interesting. This region includes the $87 \AA$ maximum and the scattering back to the (000) reflection. For the purpose of clarity, this region will be designated as very small-angle. Almost no work has been done in this region. 
Many years ago Corey and Wyckoff ${ }^{19}$ found spacings of over $100 \AA$ on the equator for the keratins; however, it was suggested by Bear ${ }^{20}$ that the origin of these spacings was somewhat questionable. It was only recently ${ }^{21,22}$ that the genuine nature of scattering in this region was confirmed for alpha keratin.

Since the $87 \AA$ maximum corresponds to the first nearest neighbor separation of microfibrils in alpha keratin, it is in this very small-angle region where the theory recently developed by Harget and Irimm ${ }^{23}$ can be applied to separate the inter-microfibrillar interference function from the intra-microfibrillar interference and hence gain some insight into the true nature of each. It should be recalled that in the past studies the intermicrofibrillar interference functions have been assumed. Wilson ${ }^{15}$ arbitrarily used three microfibrils scattering coherently, and was able to obtain fair agreement in the small-angle region. Burge, ${ }^{24}$ on the basis on the positions and the breadths of the small-angle maxima, concurred with Wilson and suggested a limited hexagonal lattice. Krimm, ${ }^{21}$ on the basis of scattering in the very small-angle region, suggested the possibility of partial cylindrical lattices. Fraser et al. ${ }^{2}$ invoked infinite coherency by using an electron micrograph combined with a radial distribution function analysis in order to obtain an inter-microfibrillar interference function for use in the small-angle and in the high-angle region. Hosemann, ${ }^{26}$ by applying paracrystalline theory to the small-angle and high-angle regions of alpha keratin (porcupine quill), was able to extract directly the paracrystalline distortion parameters. He also pointed out that a rough estimate of the paracrystallite size in the direction perpendicular to the fiber axis, based on an analysis of the undistorted meridian reflections, is of the order of $160 \AA$. Because of this rather small coherency, he suggested that the $(000)$ reflection should be analyzed to verify this estimate. Hence the problem of the proper inter-microfibrillar interference function is still open to question, but an analysis of the very small-angle region should yield the correct answer.

In the present paper we apply the methods of Harget and Krimm ${ }^{23,27}$ to the quantitative analysis of the equatorial small-angle scattering of alpha keratin. The inter-microfibrillar interference function is derived directly. The projected microfibrillar electron density is then obtained without difficulty for both native and silver-stained specimens.

\section{MATERIALS AND METHODS}

\section{Sample Preparation}

Of all the mammalian alpha keratins porcupine quill tip exhibits the most highly ordered diffraction pattern. For this reason African porcupine quill tip was used as the source of native alpha keratin for this investigation.

To study the effect of heavy metal staining, the native specimens were soaked in a $2 \% \mathrm{AgNO}_{3}$ solution for seven days, washed for one day, and subsequently vacuum dried. An attempt was made to remove the lipid by 
refluxing for seven days in 2:1 chloroform: methanol, but the net result was negative. Since a more severe chemical treatment would disrupt the protein structure, chemical extraction was abandoned and the lipid contribution was simply substracted from the diffraction pattern (see below).

\section{X-Ray Methods}

Because of the nature of the continuous scattering, the limited amount of scattering data used (470-21 $\AA$ on the equator), and the accuracy required for direct analysis of the patterns, particular care was taken to eliminate errors introduced by instrumentation. ${ }^{28}$ A Rigaku-Denki rotating anode $\mathrm{X}$-ray generator was employed. High-angle patterns were recorded using a curved Guinier camera with sample-to-film distance of $11.18 \mathrm{~cm}$ and focused copper $\mathrm{K} \alpha_{1}$ radiation. Exploratory small-angle patterns were obtained using a pinhole system, Ni-filtered copper radiation, and a sample-to-film distance of $20 \mathrm{~cm}$. Quantitative small-angle patterns were obtained using a crystal line focus system consisting of a bent quartz crystal followed by two sets of guard slits and a flat plate vacuum camera. This system gave a focused beam of copper $K \alpha_{1}$ radiation as narrow as $50 \mu$ and virtually no background or parasitic scattering, while allowing a sample-to-film distance of $20 \mathrm{~cm}$. The slit height was restricted to avoid slight smearing effects resulting from small equatorial fanning. The patterns obtained with the curved Guinier camera agreed with those obtained with the small-angle crystal line focus camera in the range $100-21 \AA$.

\section{X-Ray Analysis}

Small-angle equatorial X-ray scattering from fibrous systems derives from the combined effects of intra-microfibrillar interference, inter-microfibrillar interference, and structural inhomogeneities. Normally these effects cannot be separated, with the result that it has been necessary to rely on models or on a radial distribution function analysis to explain the scattering. Both of these approaches have serious drawbacks. Model building is subjective, tedious, and often dependent on the results of electron microscopy, which are open to question because of the rather severe preparative treatments involved. The radial distribution function analysis suffers from the fact that the microfibrillar scattering factor must be known, and when it is not, as in the case of alpha keratin, it must be assumed. The use of a radial distribution function analysis also imposes the assumptions that the scattering system is isotropic, homogeneous, and infinite in extent. These conditions are usually not satisfied in a fibrous protein system. Further, there are severe truncation errors involved in a radial distribution function analysis of paracrystalline fibrous systems. ${ }^{29}$

In order to avoid the above difficulties, a new approach to the analysis of small-angle equatorial X-ray scattering has been devised, which avoids assumptions about the scattering system. ${ }^{23.27}$ This technique relies on appropriately chosen forced intensity truncation, with a corresponding mathematical interpretation to segregate the inter-microfibrillar and the 
intra-microfibrillar interference effects. It can be applied to almost any fibrous system and yields a radius of gyration and the $z$-axis projected squared Fourier transform of the fibril, from which the $z$-axis projection of the fibril electron density can usually be obtained. In addition, it yields the inter-microfibrillar distances and the respective numbers of nearest neighbors, thus defining the geometric packing or aggregation of the microfibrils. A brief description of the technique follows.

Consider the small-angle equatorial intensity, which can be expressed as

$$
\mathrm{I}=c F^{2} \sum_{i=0}^{\bar{m}}\left\langle\frac{A_{i}}{N^{2}}\right\rangle J_{0}\left(k S_{i}\right) \exp \left[-\frac{k^{2} \sigma_{i}{ }^{2}}{2}\right]
$$

where

$$
\begin{aligned}
& k 4 \pi \sin \theta / \lambda \\
& 2 \theta \text { scattering angle } \\
& \lambda \text { X-ray wavelength } \\
& F^{2} \quad \text { Fourier transform squared of the } z \text {-axis projection of a } \\
& \text { single microfibril } \\
& \exp \left(-k^{2} \sigma_{i}{ }^{2} / 2\right] \text { lattice distortions for the vector length } S_{i} \\
& J_{0} \quad \text { zeroth order Bessel function } \\
& \bar{m} \text { maximum number of different inter-microfibrillar vector } \\
& \text { lengths present in the largest paracrystallites } \\
& S_{i} i \text { th vector length, e.g., } S_{1} \text { is the first nearest separation } \\
& \text { in real space }
\end{aligned}
$$

Note also that $S_{0}=0,\left\langle A_{0}\right\rangle=\langle N\rangle, F^{2}(0)=1$.

There is nothing dramatically new in this expression, except that it is now expressed in terms of parameters that are compatible with a Patterson function; e.g., $\left\langle A_{1} / N\right\rangle$ is the first nearest neighbor number and the $\sigma_{i}$ represent lattice distortion parameters for the $i$ th vector length $S_{i}$ in the Patterson function. This expression allows for finite crystallite size by leaving the upper limit of the sum as a parameter to be determined. The mathematical form of $F^{2}$, however, is still unknown. This is temporarily avoided by purposely truncating the intensity data at $k_{0}$ just outside, but including, the first order reflection on the equator. Then for any microfibril in this scattering region

$$
F^{2} \cong \frac{4 J_{1}^{2}\left(k R_{e}\right)}{\left(k R_{e}\right)^{2}}, \quad 0<k<k_{0}
$$


where

$R_{e}$ equivalent solid cylinder radius for the microfibril

$J_{1} \quad$ first order Bessel function.

Thus the functional form of $F^{2}$ is known in the region $0<k<k_{0}$ and involves only a single parameter $R_{e}$ which can be related to the radius of gyration of the microfibril: $R_{g}=R_{e} / \sqrt{2}$. It should be emphasized that this expression for the microfibril transform is not a model assumption but is a modification of the Guinier approximation and holds very well for $k<k_{0}<$ $\pi / R_{e}$.

It has been shown ${ }^{23}$ that when the intensity data are purposely truncated at $k_{0}$, the Patterson function can be expressed as a closed form mathematical function of the truncation points and the parameters describing the scattering system. Although this Patterson function is of low resolution, the mathematical expressions allow all the above parameters to be extracted, thus obtaining the inter-microfibrillar organization and a value for $R_{e^{2}}{ }^{27}$

\section{RESULTS}

\section{Native Alpha Keratin (African Porcupine Quill)}

The nature of the small-angle equatorial X-ray scattering from alpha keratin is illustrated in Figures 1 and 2a. In these figures the three smallangle maxima are clearly visible, but in addition there is an indication of a (000) reflection (a rise into the origin along the equator). These figures illustrate a problem encountered in attempting to obtain the $(000)$ reflection, the very low-angle region, and the low-angle region: all of the scatter-

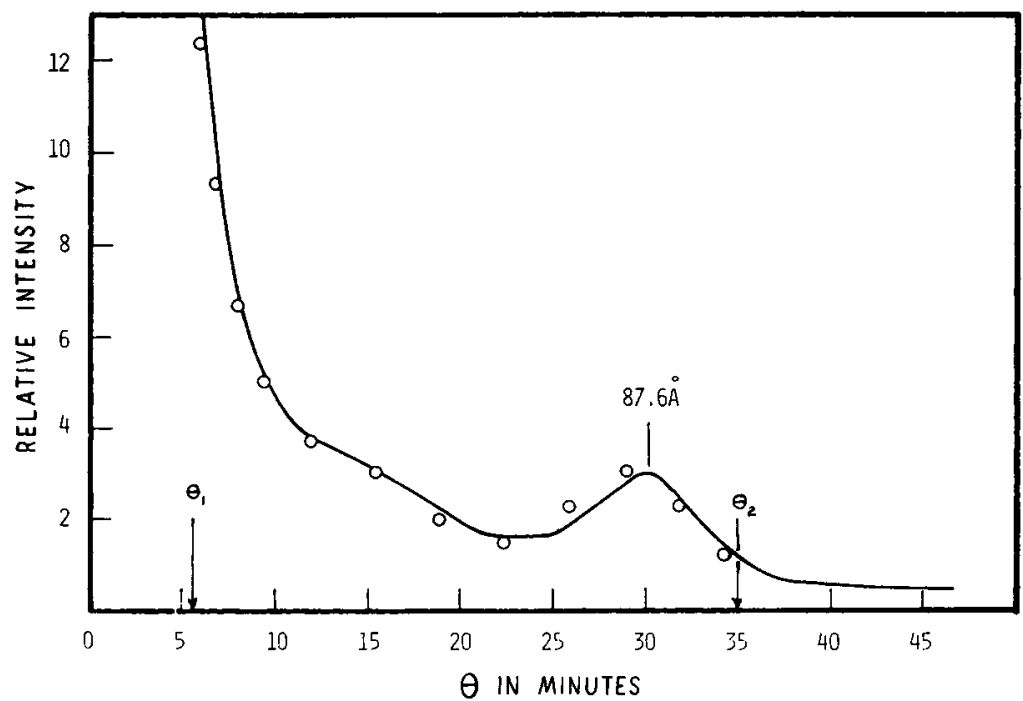

Fig. 1. Equatorial intensity for native alpha keratin (African porcupine quill) in the very small-angle region; - observed, 000 calculated. 

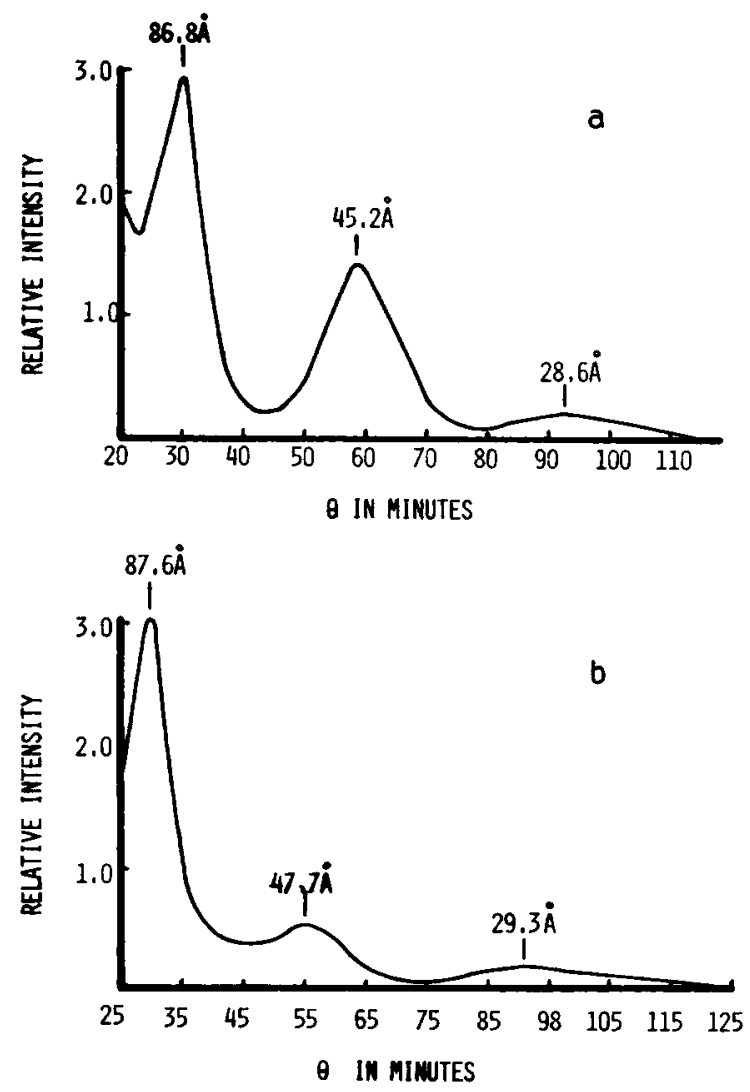

Fig. 2. Equatorial intensity for alpha keratin in the small-angle region. (a) Native. (b) Silver-stained.

ing cannot be obtained from a single exposure. We have spanned the intensity range by matching exposures on the $87 \AA$ peak, using optical densities that preserve linearity.

Such patterns showed that the first two small-angle equatorial maxima shift slightly in position as the distance of the X-ray beam from the quill tip is varied. The sharp lipid diffraction near $47 \AA$ does not shift, and this permitted it to be easily subtracted from the scattering pattern (which is the case for the curves presented here) and to help in establishing the nature of the second maximum. The two maxima appear to change affinely, for example the first one shifting from $93 \AA$ at the tip to $86 \AA$ about $3 \mathrm{~mm}$ and beyond from the tip. Quantitative data were always obtained beyond 3 $\mathrm{mm}$ from the tip. The shift in these two maxima indicates qualitatively that the first and, in part, the second maxima are caused by the intermicrofibrillar interference function.

It should be mentioned that many specimens of quill tip were used, and it was found that the nature of the $(000)$ reflection and very small-angle region did not vary from specimen to specimen. 
TABLE I

Calculated Parameters Describing the Native State of Alpha Keratin in the Very Small-Angle Region $\left(5.6^{\prime} \leq \theta \leq 35^{\prime}\right)$

\begin{tabular}{cccc}
\hline Index $i$ & $\begin{array}{c}\text { Particle } \\
\text { Separation } \\
S_{i}(\AA)\end{array}$ & $\begin{array}{c}\text { Nearest } \\
\text { Neighbor } \\
\text { Number } \\
\left\langle A_{i} / N\right\rangle\end{array}$ & $\begin{array}{c}\text { Lattice } \\
\text { Distortion } \\
\text { Parameter } \\
\sigma_{i}(\AA)\end{array}$ \\
\hline 1 & 96 & 2.12 & 6 \\
2 & 184 & 1.82 & 14 \\
3 & 270 & 0.72 & 20 \\
4 & 352 & 0.25 & 32 \\
\hline$R_{e}=33 \AA$ & \multicolumn{2}{c}{$R_{0}=23.3 \AA$} & \\
\hline
\end{tabular}

Preliminary Investigation. As suggested by Hosemann, ${ }^{26}$ who obtained a lateral paracrystallite coherent size of the order of $160 \AA$ from high-angle data, other features of the scattering pattern were checked to verify this small size of the coherently scattering domain. This was done using three different regions of the scattering pattern.

The first method involved using the lateral breadth of the third order (66 $\AA$ ) meridional diffraction line. Using the method of Bear and Bolduan ${ }^{30}$ the coherent diameter was found to be of the order of $140 \AA$. The second method involved using the region of the $(000)$ reflection which, according to the Guinier approximation, has a breadth that is reciprocally related to the coherent size. A coherent diameter of the order of $180 \AA$ was obtained using this approach. The third method involved using a half-width measurement of the first order reflection at $87.6 \AA$. Using the line broadening expression developed by Hosemann and Bagchi, ${ }^{31}$ and the lattice distortion parameter $\Delta / S_{1}=5 \%$ evaluated by Hosemann, ${ }^{26}$ a coherent size of the order of $350 \AA$ was obtained.

Although the first two methods, which derive from a two-dimensional type of calculation, involve rather crude approximations, they compare favorably with each other, and roughly with the third estimate, which derives from a one-dimensional type of calculation. The important point is that the three independent measurements indicate that a limited coherency exists. If close packing is assumed, then the number of microfibrils scattering coherently is about five to ten based on the above estimates.

Inter-Microfibrillar Organization. To solve this scattering problem in a direct quantitative manner, the Patterson function was formed using the equatorial intensity data in the following integral

$$
P\left(r, k_{1}, k_{2}\right)=\frac{\int_{k_{1}}^{k_{2}} I(k) J_{0}(k r) k d k}{\int_{k_{1}}^{k_{2}} I(k) k d k}
$$


where

$k_{1}$ lower limit of integration imposed by inaccessibility near the primary beam

$k_{2}$ upper limit of integration and can be varied.

For the alpha keratin data, $\theta_{1}=5.6^{\prime}\left(d_{1}=470 \AA\right)$. To obtain accurate values of the $S_{i}$ and to avoid the possibility of unresolved peaks, $k_{2}$ was first chosen such that $\theta_{2}=127^{\prime}\left(d_{2}=21 \AA\right)$. This allowed a resolution of $13 \AA$ in the Patterson. ${ }^{32}$ The $S_{i}$ obtained are given in Table I. To obtain the other parameters via a direct analysis of the Patterson function $k_{2}$ was chosen such that $\theta_{2}=35^{\prime}$, just outside the first order reflection on the equator. The parameters obtained are also listed in Table I.

A test of the success of any method in yielding the correct structure is to calculate the scattered intensity using the extracted parameters. This was done by putting the values given in Table $I$ into the expression

$$
I=\frac{4 J_{i}^{2}\left(k R_{e}\right)}{\left(k R_{e}\right)^{2}} \Phi
$$

where

$$
\Phi=c \sum_{i=0}\left\langle\frac{A_{i}}{N^{2}}\right\rangle J_{0}\left(k S_{i}\right) \exp \left[-\frac{k^{2} \sigma_{i}^{2}}{\mathscr{Q}}\right] .
$$

This calculated intensity is shown in Figure 1 together with the observed intensity in the region $5.6^{\prime}<\theta<35^{\prime}$. Although there is a small discrepancy on the inside shoulder of the $87.6 \AA$ peak, an effect which is caused perhaps by a small error in the measurements of the $(000)$ reflection, the agreement is quite good and the parameters listed in Table I can be taken to describe adequately the intensity in this region.

The above result has yielded an inter-microfibrillar interference function which will be used in the next section to obtain an electron density for the microfibril. This inter-microfibrillar interference function $\Phi$ is shown in Figure 3. The rapid damping of the oscillations is apparent and the oscillations become negligible past $\theta=135^{\prime}$, which is in agreement with Hosemann's estimates.

One of the first physical parameters to be extracted from the above analysis is the radius of gyration for the microfibril: $R_{g}=23.3 \AA$. Since this value is smaller than that expected from an $80 \AA$-diameter solid particle, this immediately implies that the electron density of the microfibril cannot be described as a simple solid cylinder in the small-angle region. This is all that can be said about the microfibril until the analysis of the small-angle region is completed. However all the information regarding the aggregation of the microfibrils is at hand. From Table I the value of $\left\langle A_{1} / N\right\rangle \cong 2$, which means that on the average each microfibril is surrounded by only two first neighbors. The way in which the $\left\langle A_{i} / N\right\rangle$ decrease with higher index, together with the variation of $S_{i}$ with $i$, imply that the average coherently scattering structure is an arc-like array of perhaps four to six units. These 


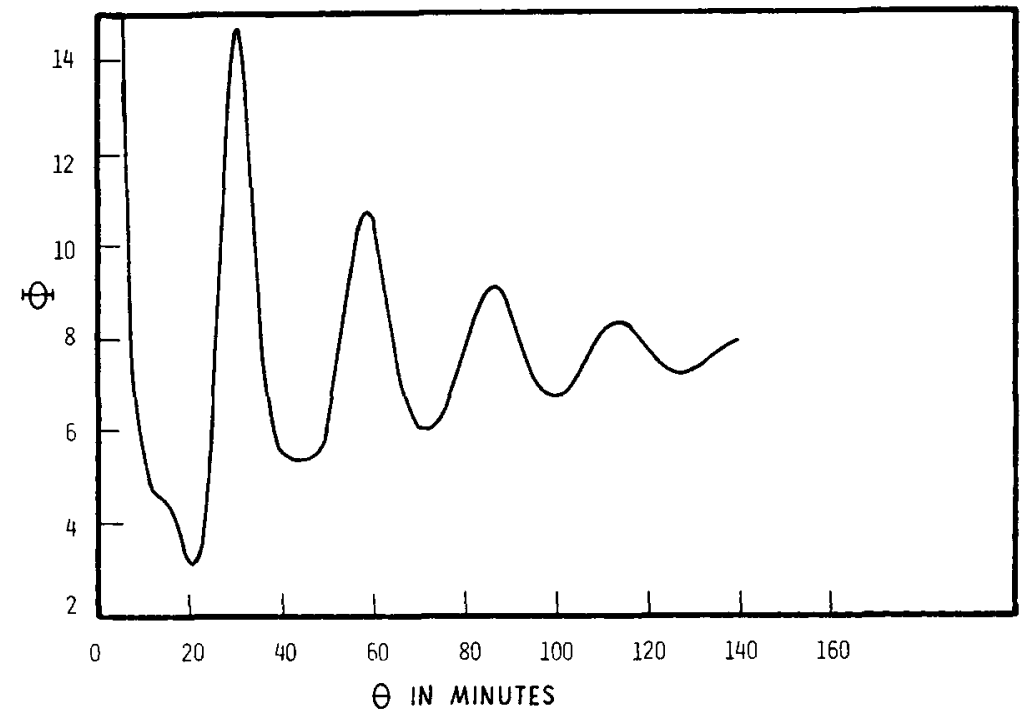

Fig. 3. Inter-microfibrillar interference function for native alpha keratin.
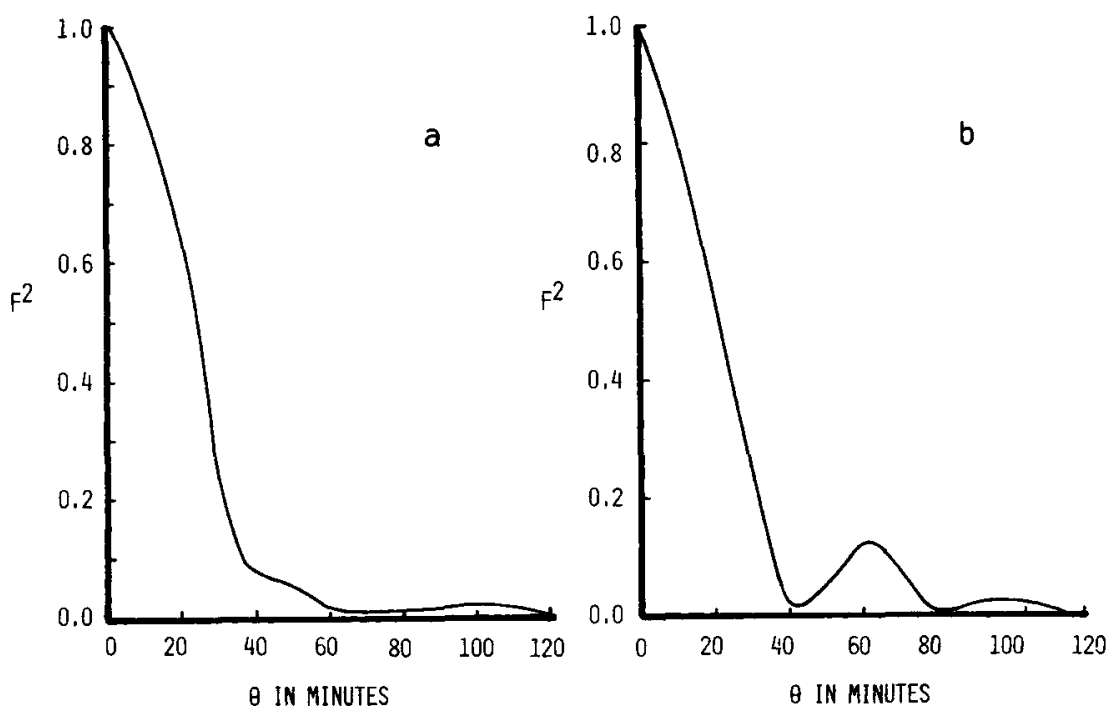

Fig. 4. Squared microfibrillar transform $F^{2}$ for alpha keratin. (a) Native.

(b) Silver-stained.

arrays probably pack roughly parallel to each other, but the ordering is poor enough so that each scatters relatively independently of the others.

The $\boldsymbol{Z}$-Axis Projection of the Electron Density. Having the inter-microfibrillar interference function, the squared Fourier transform of a single microfibril (relative to the matrix) can now be obtained to higher resolution simply by dividing the observed intensity by the intermicrofibrillar interference function $\Phi$. The resulting molecular transform squared $F^{2}$ of the microfibril is shown in Figure 4a. It is appropriate to mention the following points regarding this $F^{2}$. 
a) Although data were available only for $\theta>5.6^{\prime}, F^{2}$ was obtained to $\theta=0$ by extrapolation, since $F^{2}(0)=1$.

b) The resolution was extended only out to $\theta=127^{\prime}$ even though the inter-microfibrillar interference function past this point is approximately a constant. This is because beyond this point contributions from offequatorial components on the equator, matrix structure, etc., would have to be taken into account, and this comprises a separate problem.

c) In this region $\left(0<\theta<127^{\prime}\right)$, there is only one possible zero, located at $\theta=75^{\prime}$; hence a possible sign change could occur at this point.

To obtain the $z$-axis projection of the electron density for a cylindrically symmetric structure, the following transform was used, with $k_{2}$ corresponding to $\theta_{2}=127^{\prime}$.

$$
\rho(r)=\frac{\int_{0}^{k_{2}} \pm \sqrt{F^{2}} J_{0}(k r) k d k}{\int_{0}^{k_{2}} \pm \sqrt{F^{2}} k d k}
$$

In the region $\left(0<\theta<75^{\prime}\right)$ a positive sign was chosen, since the first sign assignment is arbitrary. (Since it is not known whether the fibril has a greater or smaller electron density than the matrix, this first sign is unknown. An error in sign here will only cause $\pm \rho(r)$; therefore this is not a serious problem.) A positive or negative sign is possible for $75^{\prime}<\theta<127^{\prime}$, but the negative sign was ruled out on the basis that the resulting $\rho(r)$ was too small in diameter $(50 \AA)$ and the structure of this $\rho(r)$ was inconsistent with the proper location of the protofibrils on the periphery.

The only other possibility therefore was the assignment of a positive sign for the entire region. The resulting $\rho(r)$ relative to the matrix is shown in Figure 5b. In Figure 5a, a schematic representation of the fine structure of a microfibril, as visualized by electron microscopy, ${ }^{4}$ is shown for convenient comparison. The calculated $\rho(r)$ has the proper overall diameter, a peak at $28 \AA$ corresponding to the location of the protofibrils on the periphery, and evidence of a core region in the microfibril.

\section{Silver-Stained Alpha Keratin}

As indicated in the last section, there are three peaks located in the smallangle region of the scattering pattern. The positions of these maxima, their relative intensities, and their shapes depend on the specimen or species from which the alpha keratin is obtained and are affected by different heavy metal stains in various ways. One of the main effects of heavy metals is to intensity this region relative to the high-angle region. In a recent paper Wilson $^{33}$ has discussed the analysis of this region in a qualitative manner, using relative intensity arguments. We describe here the quantitative results of a direct analysis of this part of the diffraction pattern.

The very small-angle region of the equatorial scattering for silverstained alpha keratin is similar to that of the native state except that there is an added component to the $(000)$ reflection. However, there are large 


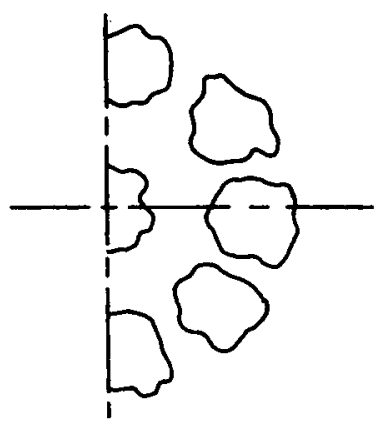

$(a)$

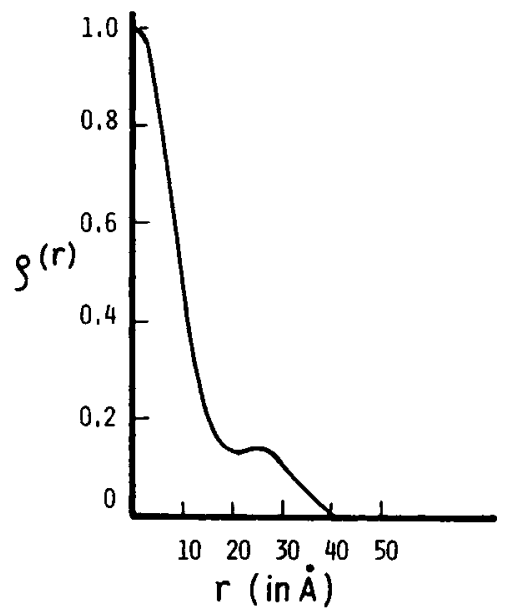

(b)

Fig. 5. (a) Schematic representation of the fine structure of a microfibril as visualized in electron microscopy. (b) Electron density for native alpha keratin, obtained to $21 \AA$ resolution.

changes in the relative peak intensities in the small-angle region, as shown in Figure $2 b$, and all three peaks are shifted slightly to lower Bragg angles. Both regions are intensified with the addition of silver.

The Patterson function was calculated using Eq. (3) with the limits of integration $7.5^{\prime}<\theta<114^{\prime}$, and the $S_{i}$ are listed in Table II. The lowresolution Patterson function was formed using the limits of integration $7.5^{\prime}$ $<\theta<35^{\prime}$ and analyzed as in the native state. As seen in Table II there are slight shifts in the vector lengths, but the nearest neighbor numbers are essentially the same as in the native state. There is also a small increase in $R_{e}$ to $36 \AA$ which implies an increase in the microfibrillar radius of gyration to $25.4 \AA$. Using these parameters an inter-microfibrillar interference function was obtained that, except for a slight shift in the positions of the peaks due to the slight shifts in the $S_{i}$, was essentially the same as that shown in Figure 3 for the native state. 


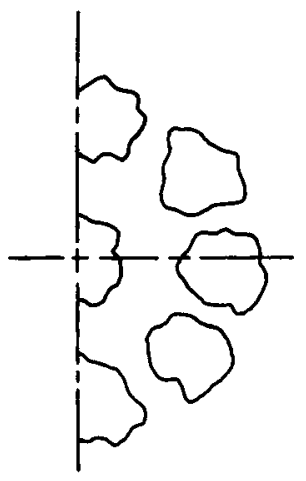

(a)

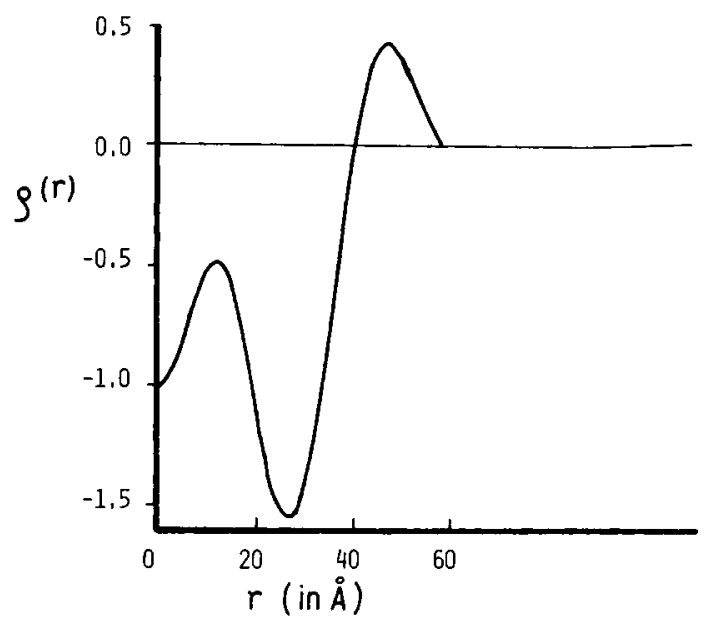

(b)

Fig. 6. (a) Schematic representation of the fine structure of a micofibril as visualized in electron microscopy. (b) Electron density for silver-stained alpha keratin, obtained to $22 \AA$ resolution.

Proceeding in a fashion similar to that for the native state, the intermicrofibrillar interference function was divided into the intensity data and the resulting $F^{2}$ is shown in Figure $4 \mathrm{~b}$.

In this $F^{2}$ there is the possibility of two zeros, at $\theta=43^{\prime}$ and $\theta=80^{\prime}$, hence the possibility of two sign changes. To obtain the $z$-axis projection of the electron density, Eq. (6) was used. The first region was arbitrarily chosen as positive; thus the following four sign combinations were possible: ,$+++++\cdot-,+-+,+--$. All four combinations were calculated. The first two were ruled out on the basis that they yiclded a $\rho(r)$ that was too large in diameter $(120 \AA)$ to be considered as being consistent with sizes measured in electron microscopy. The last one was discarded on the basis that it yielded an enormous build-up of silver at the center of the fibril, which is also inconsistent with electron microscopy. Thus the com- 
TABLE II

Inter-Microfibrillar Vector Lengths for Silver-Stained Alpha Keratin

\begin{tabular}{ccc}
\hline Index $i$ & $\begin{array}{c}\text { Particle } \\
\text { Separation } S_{i}(\AA)\end{array}$ & $\begin{array}{c}\text { Nearest Neighbor } \\
\text { Numbers }\left\langle A_{i} / N\right\rangle\end{array}$ \\
\hline 1 & 93 & 2.32 \\
2 & 182 & 1.95 \\
3 & 262 & 0.82 \\
4 & 340 & 0.35 \\
\hline$R_{e}=36 \AA$ & $R_{0}=25.4 \AA$ \\
\hline
\end{tabular}

bination +-+ is left. The $\rho(r)$ obtained with this sign combination is shown in Figure 6b, and a schematic representation of the fine structure of a microfibril, as visualized by elcctron microscopy, is shown in Figure 6a for convenient comparison.

Before discussing this $\rho(r)$, it should be mentioned that since this electron density is relative to that of the matrix, and since it is known that silver stains the matrix more heavily than the microfibrils, the electron density in Figure 6 is in the correct orientation. On this basis it is seen that silver appears to pile up slightly around the outer periphery, stains the peripheral region very little, and deposits on the inside of the peripheral region outlining the core area.

\section{DISCUSSION}

From the above analysis the fact that limited coherence exists in alpha keratin (African porcupine quill) has been firmly established and the geometry of this limited coherence quantitatively determined by the direct extraction of nearest neighbor numbers from the data. These numbers imply that the average coherent structure is primarily an arced layer-like aggregation consisting of approximately six microfibrils. This conclusion is consistent with electron micrographs such as those presented by Johnson and Sikorski:34 ares and layers are indeed predominant in porcupine quill.

Although the geometry of the coherently scattering unit has been established, the cause of this limited coherence is unknown. A possible speculation is that the microfibrils are preferentially cross-linked within the layers, thus resulting in better order between microfibrils in a given layer than between those in different layers. This idea is reinforced by the results of silver staining. Since silver is known not to stain preferentially nuclear remnants nor cell wall, ${ }^{35}$ it must be concluded that the added component of scattering in the $(000)$ reflection comes from the effect of silver intensifying the heterogeneity that caused the original limited coherence. Since the coherency did not change with the addition of silver, it appears likely that the silver deposited with a slight preference between the layers rather than within.

Further studies on various species of alpha keratin, combined with metal stains, indicate ${ }^{36}$ that although this coherency does not vary in a given 
species it does change between species. Thus insight may be gained into the nature of the matrix itself, and the manner of growth of various alpha keratins may be correlated with this coherence. In this connection Panda ${ }^{37}$ found a limited coherence in Merino wool in the very small-angle region corresponding to two different layer thicknesses.

With regard to the results obtained here for the inter-microfibrillar organization, the coherent size and lattice distortions are in good agreement with those predicted from the wide-angle diffraction pattern by Hosemann. ${ }^{26}$ Thus the use of an electron micrograph to construct an intermicrofibrillar interference function ${ }^{25}$ can give erroneous results since the limited coherency will be missed and errors will be introduced in the extracted microfibril transform and hence in the electron density constructed from the transform.

For the microfibril in the native state, the resulting $z$-axis projection of the electron density shown in Figure 5 corresponds with the concept of a peripheral and a core region. This electron density distribution automatically takes into account the protofibrils and any interstitial material without assumptions as to their nature. The figure also shows that the core region is about $20 \AA$ in diameter, the center of the periphery is located at a radius of $28 \AA$ from the center of the microfibril, the protofibrils are about $20 \AA$ in diameter, and the overall radius of the microfibril is about $40 \AA$. All of this information is consistent with electron microscopy, but it was obtained directly from equatorial X-ray data extending out to only $21 \AA$ without any model assumptions. This is to be contrasted with recent results ${ }^{18}$ in which the peripheral region was not resolved at $22 \AA$, but could only be seen when data out to $8 \AA$ were used.

There are two other points regarding the electron density of Figure 5 that need to be considered. First, because of the truncation at $21 \AA$, the resulting electron density is convoluted with the function $J_{1}\left(k_{2} r\right) / k_{2} r$, where $d_{2}=2 \pi / k_{2}=21 \AA$. However, this effect is not too serious; peaks as close as $13 \AA$ can still be resolved ${ }^{32}$ and only the shapes may be somewhat distorted. Hence the peripheral region of Figure 5 is resolved from the core region. Second, the electron density of Figure 5 is relative to the matrix located outside the microfibril. As a consequence it is not known whether this figure is right side up or upside down. Present evidence allows either possibility, and this question cannot be answered until further facts are presented.

The $z$-axis projection of the electron density in the silver-stained state shown in Figure 6 is interesting and relevant to the above question. Not only does it show approximately the same dimensions as the native state with respect to the core size, periphery location, and lateral size, but it also yields information regarding the location of the silver in and around the microfibril and some information about the microfibril itself.

Starting from the right side of Figure 6, it is seen that there is a small increase in silver concentration just outside the peripheral region. This could be due to actual binding of the silver in this region. Proceeding 
further, it is noted that the peripheral region shows a lack of stain, which is consistent with electron microscopy and the fact that stain does not penetrate the $\alpha$-helical structures, which are thought to make up the protofibrils. Then there is an increase in silver concentration between the periphery and the core region. This increase, located at about $13 \AA$, implies that the core region is not simply hollow, but must contain some material. Further, this core region may contain some structure other than simply one or two protofibrils since the minimum at the center is not as deep as the minimum located at the periphery.

There are two possibilities for the nature of this core material.

a) If the native state electron density is right side up as shown in Figure 5 , then the silver-stained result of a smaller minimum at the core follows from the fact that in the native state the core is more dense than the periphery.

b) If the native state electron density is upside down relative to that in Figure 5, then this implies that the core material must absorb more silver than the peripheral region even though silver is known to be located to some degree in the peripheral region.

Both arguments are plausible and the results of the silver-stained electron density cannot be fully utilized until the direction of the native electron density is determined. However, both a) and b) imply that there may be a difference between the core and the peripheral material. The silver treatment and analysis confirms the conjecture ${ }^{17}$ that various heavy metal treatments which cause changes in the three low-angle maxima are a result of differences cxisting both inside and outside of the microfibril.

This research was supported by U.S. Public Health Service Grant AM02830.

\section{References}

1. G. Jeffrey, J. Sikorski, and H. J. Woods, Text. Res. J., 25, 714 (1955).

2. M. Birbeck and E. Mercer, J. Biophys. Biochem. Cytol., 3, 203 (1957).

3. G. E. Rogers, Ann. N. Y. Acad. Sci., 83, 378 (1959).

4. B. Filshie and G. E. Rogers, J. Mol. Biol., 3, 784 (1961).

5. D. J. Johnson and J. Sikorski, Nature, 205, 266 (1965).

6. G. R. Millward, J. Ultrastruct. Res., 31, 349 (1970).

7. R. D. B. Fraser, T. P. MacRae, and G. R. Millward, J. Text. Inst., 60, 343 (1969).

8. R. D. B. Fraser. T. P. McRae, and A. Miller, J. Mol. Biol., 14, 432 (1965).

9. R. D. B. Fraser et al., Appl. Polym. Symp., 18, 65 (1971).

10. H. J. Woods, J. Polym. Sci., C20, 37 (1967).

11. C. Bailey and H. J. Woods, Nature, 218, 765 (1968).

12. R. D. B. Fraser and T. P. MacRae, Nature, 179, 732 (1957).

13. - - Biochim. Biophys. Acta, 29, 229 (1958).

14. —, J. Text. Inst.. T496 (1960).

15. H. R. Wilson, J. Mol. Biol., 6, 474 (1963).

16. C. Tyson and H. J. Woods, J. Mol. Biol., 9, 266 (1964).

17. H. J. Woods and C. Tyson, Nature, 209, 399 (1966). 
18. R. D. B. Fraser, T. P. MacRae, and D. A. D. Parry, Symp. Fibrous Proteins Australia 1967, W. G. Crewther, Ed., Butterworths, Sydney, Australia, p. 279.

19. R. B. Corey and W. G. Wyckoff, J. Biol. Chem., 114, 407 (1936).

20. R. S. Bear, J. Amer. Chem. Soc., 66, 2043 (1944).

21. S. Krimm, in Small-Angle X-Ray Scattering, H. Brumberger, Ed., Gordon and Breach, New York, 1967.

22. P. J. Harget and S. Krimm, presented at the 3rd Internat. Biophys. Congr., Cambridge, Mass., 1969.

23. - Acta Cryst., A27, 586 (1971).

24. R. E. Burge, J. Mol. Biol., 7, 213 (1963).

25. R. D. B. Fraser, T. P. MacRae, A. Miller, and E. Suzuki, J. Mol. Biol., 9, 250 (1964).

26. R. Hosemann, Acta Cryst., 4, 520 (1951).

27. P. J. Harget and S. Krimm, J. Polym. Sci. (A-2), 10, 919 (1972).

28. P. J. Harget, Norelco Rept., 18, 25 (1971).

29. - - Ph.D. dissertation, University of Michigan, 1969.

30. R. S. Bear and O. E. A. Bolduan, Acta Cryst., 3, 236 (1950).

31. R. Hosemann and S. N. Bagchi, Direct Analysis of Diffraction by Matter, NorthHolland, Amsterdam, 1962.

32. R. W. James, Optical Principles of the Diffraction of X-Rays, Bell, London, 1948.

33. G. A. Wilson, Polymer, 13, 63 (1972).

34. D. J. Johnson and J. Sikorski, Nature, 194, 31 (1962).

35. M. G. Dobb, J. A. Nott, and J. Sikorski, Eur. Reg. Conf. El. Micr., 2, 664 (1960).

36. P. J. Harget, S. Krimm, and J. Stamatoff, presented at the 14th Biophys. Soc.

Meeting, Baltimore, Md., 1970.

37. B. C. Panda, Brit. J. Appl. Phys., 3, 815 (1970).

Received May 24, 1973

Revised October 1, 1973 